\title{
Night-time Vehicle Detection for Automatic Headlight Beam Control
}

\author{
Pushkar Sevekar \\ Student, Department of \\ Electronics Engineering \\ A.I.S.S.M.S. Institute of \\ Information Technology, \\ Pune, India
}

\author{
S. B. Dhonde \\ Associate Professor, \\ Department of \\ Electronics Engineering \\ A.I.S.S.M.S. Institute of \\ Information Technology, \\ Pune, India
}

\begin{abstract}
A clear visibility of the road ahead is significant concern for safe nighttime driving. However, high beams are utilized less than sufficient on the roads since drivers are afraid of dazzling others. Subsequently, the smart programmed control of vehicles front lamp is of incredible significance.

According to road accident surveys, majority of the accidents occur in dark. Visibility in dark is significant issue for safe driving. Therefore careless drivers continue using a high beam even though approaching vehicle is observed. These high beams create glare for approaching drivers which then causes temporary blindness. To solve this problem, nighttime vehicle detection has a great importance. This paper reviews various attempts made to solve the problem, need of study, currently implemented relevant systems and related work, different approaches to solve problem and various applications. The survey shows sensor based approaches cannot be used to satisfy real time requirements of a system. However, a simple and reliable solution needs to be developed so that, it can be implemented in each vehicle. An attempt has been made to present an image processing based system that detects vehicles and selects ideal beam so that accidents due to temporary blindness can be reduced.
\end{abstract}

\section{General Terms}

Light blob detection, Support vector machines.

\section{Keywords}

Headlight control, Nighttime vehicle detection, beam control, Light blob detection, Low beam, High beam.

\section{INTRODUCTION}

Road transportation has offered many advantages both to society and to people by providing movements of goods and individuals and making easy access to various social and economic services. The quick rise in motorization along with expansion of road network has brought with it the challenge of facing adverse factors such as the increase in road accidents.Even with the fact that having lower traffic volume, 42 percent of all traffic accidents occur after dark, whereas 58 percent are fatal accidents and 67 percent are pedestrian casualties [1]. Therefore vehicle detection during night time holds a great importance for implementation of safety features in vehicles.

ADAS (Advanced Driver Assistance Systems) which assist driver while driving have been a popular research focus over the past few years. These systems provide various assistance functions such as Autonomous cruise control, Blind Spot Information System, Cooperative Adaptive Cruise Control, Lane departure warning, forward collision warning, Adaptive
Front-lighting System, Pedestrian Collision Warning etc. Many of these systems are implemented to their basic functionality whereas some of them are being tested. [2]

To improve road illumination and reduce accidents at night, identification of vehicle in front of ego vehicle as well as approaching vehicles (travelling in the other direction than a host vehicle) is necessary. Detecting vehicles based on their appearance features such as color, shape or typical pattern is feasible during day time. For night time detection of vehicles, headlights and tail lights are the most reliable features.

\section{RELATED WORK AND IMPLEMENTATIONS}

Night time vehicle detection is a vast area for research. Such research is useful in driver assistance systems as well as traffic surveillance systems. Many researchers have presented their work in the subject. Some applications use the nonvision based approaches [3],[4] to implement driver assistance systems whereas others use vision based approaches for traffic surveillance and driver assistance systems [5],[6],[7]-[13]. Headlights have been widely used for detecting vehicles at night. Nighttime detection of vehicle has many applications in traffic monitoring systems and driver assistance systems.

This chapter gives an outline of state-of-the-art methods proposed by researchers and relevant systems developed by automobile manufacturers. Some of the headlight control systems developed currently are multi beam LED system by Mercedes Benz, Smart beam by Gentex Corporation and AHC by mobil-eye.

- New multibeam LED technology by Mercedes Benz [14]. This system is being incorporated first time into the new-generation CLS-Class cars. They have grid pattern light source comprised of 24 individual LED chips. These chips are independently controlled in order to obtain continuous non dazzling high beam. However, absence of low beam in such systems becomes problem due to legal regulation in some countries.

- Gentex corporation developed a SmartBeam ${ }^{\mathrm{TM}}$ [15] for detecting headlight and taillight and automatically controlling headlight beam. The system is built upon a CMOS image sensor for capturing images and a controller to generate headlight control signals.

- Adaptive headlight control (AHC) developed by mobil-eye [16] implements a similar function using camera and detailed analysis of light sources in an image. AHC allows drivers to use high beam as 
much as possible and also considers the scenario of urban/lit area.

In vision based approach, camera mounted on a room mirror inside windshield captures images. These images are then processed to detect headlight blobs present in image.

Sungmin Eum et al (2013) proposed a system with the images captured in two different settings namely low exposure and auto exposure using a single camera module. A fixed thresholding is applied to low exposure images whereas LoG filtering is applied to auto exposure images so that significant features of images can be obtained. These results are integrated for light blob detection. After detection of headlights, kalman filtering algorithm is used for tracking. Tracking is then followed by classification of blob into headlight, taillight, street light or nuisance light using a pattern recognition based classifier.

P. F. Alcantarilla et al (2011) proposes a detection method with an adaptive thresholding. After detection of bright objects in an image clustering of the blobs is done with respect to their geometric characteristics. Clustering is useful for classification of bright object in an image as vehicle or nuisance light. For tracking of cluster this method also used kalman filter, whereas for classification support vector machine based classifier is used.

Another approach to deal with headlight glare is presented in [3]. This method uses light intensity sensors for detection of oncoming vehicle, whereas after detection of excess light, a request is sent to an oncoming vehicle to reduce its headlight intensity. Similar attempt is shown in [4] but this method lowers a beam of ego vehicle instead of reducing intensity of oncoming vehicle.

\section{METHODOLOGY}

Researchers have used mainly two approaches in order to deal with headlight glare and temporary blindness. This section discusses these approaches in detail. The most common approach is by using a camera to capture images in nighttime. This image sequence is then processed in order to detect presence of vehicle. Another approach to deal with this problem of temporary blindness is by using light intensity sensor and estimation of presence of vehicle based on intensity of light.

\subsection{Image based approach}

A general flow for image based approach follows four main steps. These steps are light detection, pairing, tracking and classification [7].
- Headlight identification: Various approaches for detecting headlight blob are based on thresholding techniques. The most common among them is fixed thresholding [5][8],[9]or adaptive thresholding[6]. Though thresholding is fast, it is incapable of capturing objects structure. This leads to use of LoG filters [5]. The result of these methods is extracted light blob.

- Pairing: Pairing is helpful for recognizing vehicles from other reflections. If a blob of similar size, shape etc. is found in neighborhood, it s highly possible that they are from same vehicle. This is an optional step as suggested by [9] since there can be more than one blob with similar characteristics in neighborhood or two blobs of a same vehicle may not be similar at times.

- Tracking: Tracking provides motion data and hence is useful in identifying vehicles at nighttime. For nighttime detection, headlights are tracked. The very common approach is using kalman filter [5],[6],[7]. Other forms of tracking are rule based tracking [10] and nearest neighbor matching [11].

- Classification: Reflection from non light sources and street light can often cause trouble for vehicle detection. This problem is solved by different methods such as rule based methods [12], physical model based method [13], and machine learning methods [6].

\subsection{Sensor based approach}

This method uses a light intensity sensor in order to detect presence of incoming traffic in night scene. Sensor is usually a photo sensitive device, LDR is used in [3], [4] for detecting light intensity. There is a linear relation among sensor output and light intensity. Outputs of sensor are converted into digital form so as to compare them against threshold. Threshold is useful to identify presence of vehicle and it is fixed at a point where abrupt intensity change occurs.

In [3] headlight glare from oncoming vehicle is notified to that vehicle. This is done using xbee module, 


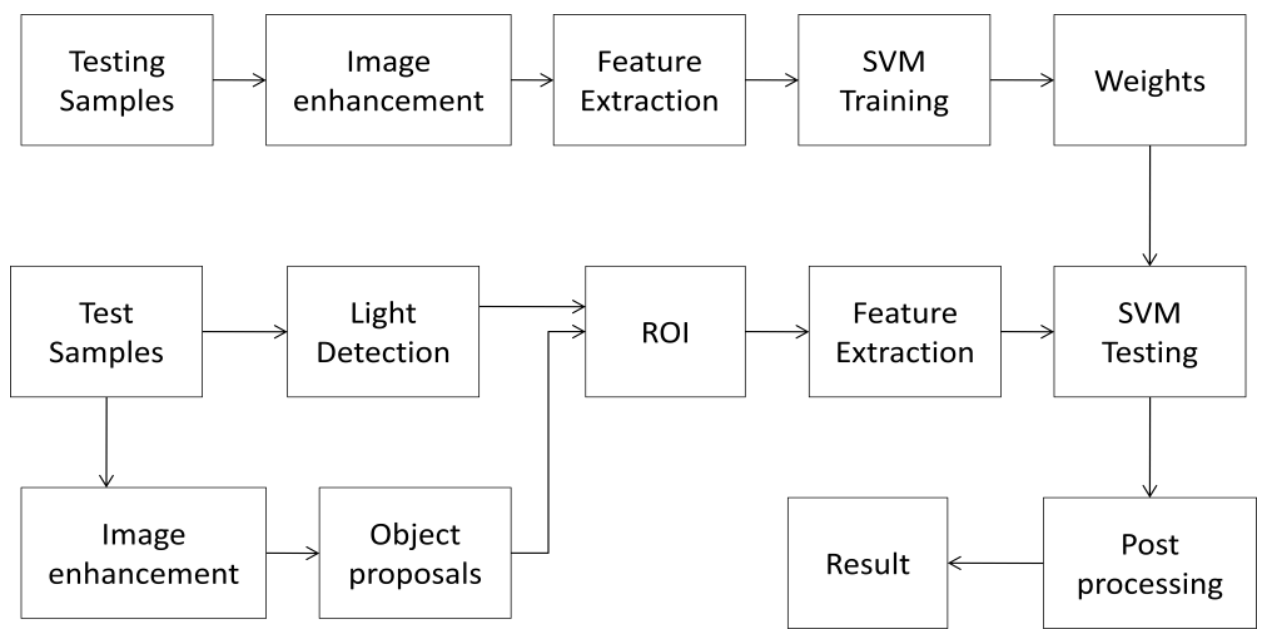

Figure 1. Block diagram for nighttime vehicle detection

such communication is used to reduce incoming light intensity.

Considering effectiveness of camera over light intensity sensors, Image based approach for night time vehicle is selected. The proposed approach for detection of vehicles is shown in figure 1 .

\subsubsection{ROI extraction}

For extracting regions of interest (ROI), contrast enhancement of an input image is performed. For this purpose, RGB image is converted into color intensity image as,

$$
C i=\frac{\operatorname{Max}(R, G, B)}{255}
$$

For noise reduction and contrast enhancement between vehicle lights and background, color intensity image is filtered using step function. To emphasize vehicle lights, nakagami image [17] is computed from color intensity image. Nakagami image is now converted to binary image for locating light regions using counter detection. Bounding boxes are obtained on enhanced image for generating object proposals. Finally, with the help of vehicle light regions and object proposals ROI's are computed.

\subsubsection{Feature extraction}

This method uses five features: local binary pattern (LBP) [18], histogram of oriented gradients (HOG) [19] four direction features (FDF) [20], HSV color histogram (named color), and edgeHOG (HOG based on structured edges in [21]), The five features represent different attributes of the vehicle and demonstrate different accuracy for vehicle recognition. Feature vectors of each feature are used for SVM training.

\subsubsection{Classification}

This stage performs classification of objects in vehicles and non vehicles. Support vector machines are used for classification. Initially support vector machines are trained using feature vectors of positive and negative samples. To improve recognition accuracy, combination of scores of each feature along with their weights is performed. Classification contribution is dependent on weight of each classifier.

\section{EXPERIMENTAL RESULTS}

In order to estimate effectiveness of proposed method, several night scene images were evaluated with proposed algorithm. Performance is measured by determining classification accuracy, detection rate, miss rate and false positives per image.

\subsection{Classifier Performance}

Classifier training was performed for each feature extracted. For finding classification accuracy, 200 images containing positives and negatives were given to each classifier Depending on the accuracy of each feature; weights for each feature are computed so as to use them during testing phase. Detection performance for each classifier is as follows

Table 1. Classification accuracy

\begin{tabular}{|c|c|}
\hline Feature & Accuracy \\
\hline FDF & $87 \%$ \\
\hline edgeHOG & $73 \%$ \\
\hline HSV & $90.50 \%$ \\
\hline LBP & $76 \%$ \\
\hline HOG & $90.50 \%$ \\
\hline Fusion & $94.50 \%$ \\
\hline
\end{tabular}

\subsection{Simulation Results}

Test was conducted on 243 nighttime image samples for detection of vehicle presence and identifying ambient light sufficiency to declare ideal headlight beam. It is seen that, proposed algorithm is able to detect presence of vehicle in most cases. However, depending upon night scene complexity, false positives are observed. Examples of correct detection and false positives are shown below.

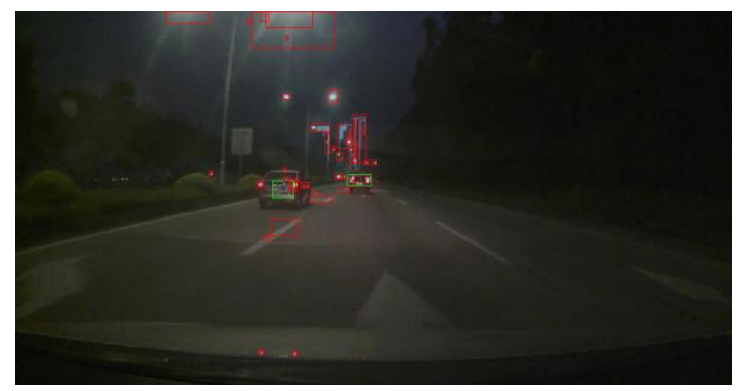

Figure 2 Correct detection 


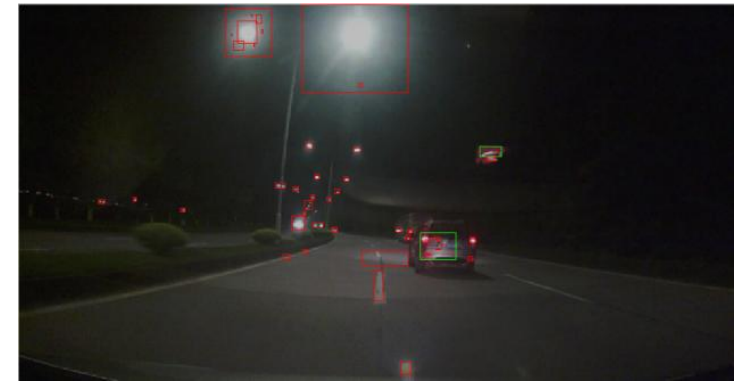

Figure 3 False positive due to infrastructure elements

\subsection{Detection Results}

For proposed method, detection rate, false positives per images and miss rate are significant performance parameters. Detection rate is computed as a ratio of correctly detected vehicles to the total number of ground truths. Mathematically expressed as,

$$
\begin{aligned}
\text { Detection Rate } & (\%) \\
& =\frac{\text { Correct Detections }}{\text { Ground truths }} \\
& \times 100
\end{aligned}
$$

However, false positives per image are computed as the ratio of total false positives obtained to the total number of samples in evaluation dataset. Mathematically expressed as,

$$
F P P I=\frac{\text { Total false detections }}{\text { Number of images }}
$$

Miss rate is computed as, ratio of total number of missed detection to the number of image samples in dataset. Table 2 shows results for experimental analysis. However, detection rate for which is $94.84 \%$, false positives per image is 0.156 and miss rate is 0.617

\section{Table 2. Test results}

\begin{tabular}{|c|c|c|c|}
\hline \multicolumn{4}{|c|}{ Total samples (243) } \\
\hline $\begin{array}{c}\text { Ground } \\
\text { Truths }\end{array}$ & $\begin{array}{c}\text { Correct } \\
\text { Detections }\end{array}$ & $\begin{array}{c}\text { False } \\
\text { positives }\end{array}$ & $\begin{array}{c}\text { Missed } \\
\text { detection }\end{array}$ \\
\hline 291 & 276 & 38 & 15 \\
\hline
\end{tabular}

\section{CONCLUSION}

In this paper a method for intelligent headlight control is presented. Many researchers have worked in area of nighttime vehicle detection for headlight control. Some automotive manufacturers have implemented similar systems into their high end vehicles.

Nighttime vehicle detection is still an open area for research since, a cost friendly solution to this problem can only incorporate such system into low budget automotive. However, this attempt of accurate detection addresses issue of temporary blindness well and provides a reliable algorithm for headlight control. Further studies can be focused to improvising detection range, false positives per image. Making system more portable so that it can be mounted on two wheelers is also in interest of practical headlight beam control systems. Analysis of unique features of headlights and tail light so as to improve detection rate is scheduled for further study. The idea of detecting vehicles at night time is useful for various applications such as, traffic surveillance, smart headlight beam control, lane departure warning etc.
Such functions can be merged together in future to obtain advanced driver assistance systems.

\section{ACKNOWLEDGMENTS}

We would like to thank Dr. P. B. Mane for their support during this work and for giving timely suggestions while the process of the work. We are also thankful to department of electronics AISSMS IOIT pune for their support.

\section{REFERENCES}

[1] New Headlight Sensors Make Night Driving Safer, Road and Travel Magazine, 2007. [Online]. Available: http://www.roadandtravel.com/autoadvice/2007/highbea ms.htm

[2] Feng Luo And Fengjian $\mathrm{Hu}$, A Comprehensive Survey Of Vision Based Vehicle Intelligent Front Light System, International Journal On Smart Sensing And Intelligent Systems Vol. 7, No. 2, June 2014, pp. 701-723.

[3] Amiya Kumar Tripathy, Deepali Kayande, Joel George, Jerome John, Bejoy Jose, Wi Lights - A Wireless Solution To Control Headlight Intensity, IEEE International Conference on Technologies for Sustainable Development (ICTSD-2015), Feb. 04-06, 2015, Mumbai, India

[4] Mohammed Alsumady and Shadi. A. Alboon, Intelligent Automatic High Beam Light Controller, (02013 Old City Publishing, Inc. J. of Active and Passive Electronic Devices, Vol. 00, pp. 1-8.

[5] Sungmin Eum and Ho Gi Jung, Enhancing Light Blob Detection for Intelligent Headlight Control Using Lane Detection, IEEE TRANSACTIONS ON INTELLIGENT TRANSPORTATION SYSTEMS, VOL. 14, NO. 2, JUNE 2013, pp 1003-1011

[6] P. F. Alcantarilla, Automatic LightBeam Controller for driver assistance, Springer-Verlag Machine Vision and Applications September 2011, Volume 22, Issue 5, pp 819-835

[7] Darko Juri'c and Sven Lon cari'c, A Method for On-road Night-time Vehicle Headlight Detection and Tracking, IEEE Connected Vehicles and Expo (ICCVE), 2014 International Conference Vienna 3-7 Nov. 2014 , pp 655 -660 .

[8] Y. Li, N. Haas, and S. Pankanti, "Intelligent headlight control using learning-based approaches," in Intelligent Vehicles Symposium (IV),2011 IEEE. IEEE, 2011, pp. 722-727.

[9] A. L'opez, J. Hilgenstock, A. Busse, R. Baldrich, F. Lumbreras, and J. Serrat, "Nighttime vehicle detection for intelligent headlight control," in Advanced Concepts for Intelligent Vision Systems. Springer, 2008, pp. 113124.

[10] Y.-L. Chen and C.-Y. Chiang, "Embedded vision-based nighttime driver assistance system," in Computer Communication Control and Automation (3CA), 2010 International Symposium on, vol. 2. IEEE, 2010, pp. 199-203.

[11] A. Fossati, P. Sch'onmann, and P. Fua, "Real-time vehicle tracking for driving assistance," Machine Vision and Applications, vol. 22, no. 2, pp. 439-448, 2011.

[12] R. O'Malley, E. Jones, and M. Glavin, "Rear-lamp vehicle detection and tracking in low-exposure color 
video for night conditions," IEEE Trans.Intell. Transp. Syst., vol. 11, no. 2, pp. 453-462, Jun. 2010.

[13] W. Zhang, Q. M. J. Wu, G. Wang, and X. You, "Tracking and pairing vehicle headlight in night scenes," IEEE Trans. Intell. Transp. Syst., vol. 13, no. 1, pp. 140153, Mar. 2012.

[14] Multibeam LED brings light into the darkness [Online]. Available:

https://www.mercedesbenz.com/en/mercedes-benz/innovation/multibeam-ledbrings-light-into-the-darkness/

[15] Lighting Assist - SmartBeam® ${ }^{\circledR}$ [Online]. Available: https://www.gentex.com/automotive/products/forwarddriving-assist

[16] Mobileye Binary Headlamp Contol [Online]. Available: http://www.mobileye.com/technology/applications/headlamp-control/binary-headlamp-contol/
[17] D.-Y. Chen et al., "Nighttime Brake-Light detection by nakagami imaging," IEEE Trans. Intelligent Transportation Systems, vol. 13, no. 4, pp. 1627 - 1637, 2012.

[18] T. Ahonen et al., "Face recognition with local binary patterns," in Proc.European Conference on Computer Vision, 2004, pp. 469-481.

[19] N. Dalal and B. Triggs, "Histograms of oriented gradients for human detection," in Proc. IEEE Conf Computer Vision and Pattern Recognition, vol. 1, no. 12, 2005, pp. 886-893.

[20] H. Kuang et al., "MutualCascade method for pedestrian detection," Neurocomputing, vol. 137, pp. 127-135, 2014.

[21] P. Doll'ar and C. L. Zitnick, "Fast edge detection using structured forests," IEEE Trans. Pattern Analysis and Machine Intelligence, vol. 37,no. 1, pp. 1-1, 2015. 